\title{
HOLOMORPHIC CURVES IN ALGEBRAIC MANIFOLDS ${ }^{1}$
}

\author{
BY BERNARD SHIFFMAN
}

A holomorphic curve in a complex manifold $M$ is a nonconstant holomorphic map $f: C \rightarrow M$. In 1927, R. Nevanlinna [18] created a new theory concerning the distribution of values of a holomorphic curve $f$ in the complex projective line $\mathbf{C P}^{1}$. Nevanlinna's main result is that $f$ assumes almost all values in $\mathbf{C P}^{1}$ "equally often," and those values that $f$ fails to assume often enough have total "defect" at most 2. H. Cartan [7] generalized this "defect relation" to holomorphic curves $f$ in $\mathbf{C P}^{n}$, counting how often $f$ takes values in hyperplanes; L. Ahlfors [2] later reproved and extended Cartan's result, which he cast in a geometric form. Recently, J. Carlson and P. Griffiths [6] obtained new defect relations for holomorphic maps $f: \mathbf{C}^{n} \rightarrow M$, where $M$ is an $n$-dimensional algebraic manifold, counting how often $f$ takes values in divisors of a fixed holomorphic line bundle on $M$. Nevertheless, except for the results of Nevanlinna, Cartan and Ahlfors, very little is presently known about the distribution of values of holomorphic curves.

The first three sections describe the results of R. Nevanlinna [18], Cartan [7] and Ahlfors [2], and Carlson and Griffiths [6], respectively. (H. Cartan's brief, but not very well-known, proof of the defect relation for holomorphic curves in $\mathbf{C P}^{n}$ is given in \$2.) $\$ 4$ states some open problems on the distribution of values and existence of holomorphic curves in algebraic manifolds, as well as giving some recent results of R. Brody and of M. Green [4], [5], [14]. Finally, $\$ 5$ gives a new proof of the Cartan-Ahlfors defect relation, which places it within the geometric framework of Carlson and Griffiths [6].

Other recent expositions of value distribution theory, which concentrate on mappings of several complex variables, are contained in the monograph by $P$. Griffiths [27] and the survey article by W. Stoll [29].

I learned about value distribution theory from Phillip Griffiths, Reese Harvey, and Yum-Tong Siu, whom I wish to thank for sharing their insights with me.

1. Nevanlinna theory. Let $f$ be a nonconstant meromorphic function on the complex line $\mathbf{C}$. We regard $f$ as a holomorphic curve in the complex projective line $\mathbf{C P}^{1}$ by identifying $\mathbf{C P}^{1}$ with the Riemann sphere $\mathbf{C} \cup\{\infty\}$. The earliest result on the values of $f$ is Liouville's Theorem (1847) that $f$ cannot omit an open set in $\mathbf{C P}^{1}$; i.e., $f(\mathbf{C})$ is dense in $\mathbf{C P}^{1}$. In 1879, Picard improved this

This is an expanded version of an invited address given at the Cambridge, Massachusetts, meeting of the American Mathematical Society on October 25, 1975; received by the editors May 20, 1976.

AMS (MOS) subject classifications (1970). Primary 32-02, 32H99, 32H25, 30A70; Secondary $32 \mathrm{H} 20$.

'This work was partially supported by NSF Grant GP-40931 and by a Sloan Fellowship. 
result by proving that $f$ can omit at most two values in $\mathbf{C P}^{1}$, and in fact if $f$ is transcendental, then $f$ attains all but at most two values infinitely many times (Picard's proof uses the uniformization of $\mathbf{C}-\{0,1\}$ by the upper half plane). In 1896, E. Borel [3] gave a new proof of Picard's Theorem using the concept of the rate of growth of an entire function. In 1927, R. Nevanlinna [18] refined Borel's idea and developed a new branch of complex function theory culminating with the Nevanlinna Defect Relation (see (1.11)), which substantially sharpens Picard's Theorem.

We now describe Nevanlinna's elegant theory of meromorphic functions on the complex line. Let $r_{0}>0$ be fixed. We let

$$
t_{f}(r)=\int_{\Delta(r)} f^{*} \omega
$$

where $\omega=(\sqrt{-1} / \pi) \partial \bar{\partial} \log \|w\|\left(w=\left(w_{0}: w_{1}\right) \in \mathbf{C P}^{1}\right)$ is the "spherical" area form on $\mathbf{C P}^{1}$ normalized so that $\int \omega=1$, and $\Delta(r)=\{z \in \mathbf{C}:|z|<r\}$. I.e., $t_{f}(r)$ is the volume of $f(\Delta(r))$ counted with multiplicities. The order function of $f$ is given by

$$
T_{f}(r)=\int_{r_{0}}^{r} t_{f}(s) s^{-1} d s, \text { for } r \geqslant r_{0} .
$$

(The above definition of the Nevanlinna order function is due to Ahlfors [1] and Shimizu [22] and agrees with Nevanlinna's definition modulo a bounded term.) We shall use the notation

$$
M_{r} \phi=\frac{1}{2 \pi} \int_{0}^{2 \pi} \phi\left(r e^{i \theta}\right) d \theta
$$

By integrating (1.2) by parts and then applying Stokes' theorem, we obtain the alternate description of the order function,

$$
T_{f}(r)=M_{r} \log \|\tilde{f}\|-M_{r_{0}} \log \|\tilde{f}\|,
$$

where $\tilde{f}: \mathbf{C} \rightarrow \mathbf{C}^{2}-\{0\}$ represents $f$ (i.e., $f=\left(\tilde{f}_{0}: \tilde{f}_{1}\right)$ ). We say that $f$ is of finite order if $T_{f}(r) \leqslant r^{\lambda}+O(1)$ for some finite $\lambda$. Let $a \in \mathbf{C P}^{1}$ be arbitrary. We let $n_{f}(a, r)$ denote the number of solutions of $f=a$ (counted with multiplicities) in $\Delta(r)$. The counting function $N(a, r)$ is given by

$$
N(a, r)=N_{f}(a, r)=\int_{r_{0}}^{r} n_{f}(a, s) s^{-1} d s, \text { for } r \geqslant r_{0} .
$$

It follows from the above that

$$
T_{f}(r)=\int_{\mathbf{C P}^{1}} N(a, r) \omega(a)
$$

i.e., $T_{f}(r)$ is the average value of $N(a, r)$.

Nevanlinna's First Main Theorem (see (2.6)) tells us that

$$
N(a, r) \leqslant T_{f}(r)+O(1)
$$

for all $a \in \mathbf{C P}^{1}$. We shall let $S_{f}(r)$ be the symbol for any function on $\left(r_{0},+\infty\right)$ with the property that

$$
S_{f}(r) \leqslant O\left(\log r T_{f}(r)\right) \text { for } r \notin E,
$$


for some set $E \subset\left(r_{0},+\infty\right)$ of finite length. (In fact, we can require that

$$
S_{f}(r) \leqslant O\left(\log T_{f}(r)\right)+o(\log r) \text { for } r \notin E ;
$$

see [21, p. 169]. If $f$ is of finite order, we can require that (1.8) hold without exceptional intervals; i.e., $S_{f}(r) \leqslant O(\log r)$ for all $r>r_{0}$.) Nevanlinna's deep result is his Second Main Theorem

$$
(q-2) T_{f}(r) \leqslant \sum_{j=1}^{q} N\left(a_{j}, r\right)+S_{f}(r),
$$

where $\left\{a_{1}, \ldots, a_{q}\right\}$ is any collection of distinct points in $\mathbf{C P}^{1}$. The Nevanlinna defects $\delta(a)$ are defined by

$$
\delta(a)=\liminf _{r \rightarrow+\infty}\left[1-\frac{N(a, r)}{T_{f}(r)}\right] .
$$

It follows from (1.9) that

$$
\sum \delta(a) \leqslant 2,
$$

where the summation is over all $a \in \mathbf{C P}^{1}$. Equation (1.11) is the well-known Nevanlinna Defect Relation, which implies Picard's Theorem since $\delta(a)=1$ if $f$ is transcendental and $f^{-1}(a)$ is a finite set.

EXAMPLES. (i) Let $f(z)=e^{z}+e^{\lambda z}$, where $0<\lambda \leqslant 1$. Then

$$
T_{f}(r)=r / \pi+O(1), \quad \delta(\infty)=1, \quad \delta(0)=\lambda,
$$

and all the other defects are zero (see [2]).

(ii) Let $f(z)=\int_{0}^{z} e^{-t^{q}} d t$, where $q$ is a positive integer. Then

$$
\begin{aligned}
& T_{f}(r)=[1+o(1)] r^{q} / \pi, \\
& \delta(\infty)=1, \quad \delta\left(c_{q} e^{2 \pi i / k}\right)=1 / q, \quad \text { for } 1 \leqslant k \leqslant q,
\end{aligned}
$$

where $c_{q}=\int_{0}^{+\infty} e^{-x^{q}} d x$, and all the other defects are zero (see [18, p. 21]).

Nevanlinna's Second Main Theorem (1.9) is a consequence of the following deep result:

(1.12) Lemma on the logarithmic derivative [18]. Let $\mathrm{g}: \mathbf{C} \rightarrow \mathbf{C} \cup\{\infty\}$ be a nonconstant meromorphic function. Then

$$
M_{r} \log ^{+}\left|g^{\prime} / g\right| \leqslant S_{g}(r) .
$$

We use the notation $\log ^{+} x=\max (\log x, 0)$. In fact Lemma (1.12) is the crux of value distribution theory, and all results in the subject use this lemma either directly or implicitly. It follows from (1.12) that

$$
M_{r} \log ^{+}\left|g^{(m)} / g\right| \leqslant S_{g}(r)
$$

for $m=1,2,3, \ldots$.

2. Holomorphic curves in $\mathbf{C P}^{n}$. We next discuss the Cartan-Ahlfors generalization of the Second Main Theorem (1.9) to holomorphic curves in $\mathbf{C P}^{n}$, and we give Cartan's brief proof, which utilizes the lemma on the logarithmic derivative. Let $w=\left(w_{0}: \ldots: w_{n}\right)$ denote the homogeneous coordinates in $\mathbf{C P}^{n}$. We let 


$$
\omega=(\sqrt{-1} / \pi) \partial \bar{\partial} \log \|w\|
$$

denote the Kähler form (of the Fubini-Study metric) on $\mathbf{C P}^{n}$, normalized so that $\omega$ represents the positive generator of $H^{1}\left(\mathbf{C P}^{n}, Z\right)$.

Let $f: \mathbf{C} \rightarrow \mathbf{C P}^{n}$ be a holomorphic curve such that $f(\mathbf{C})$ is not contained in a hyperplane of $\mathbf{C P}^{n}$. We define the order function $T_{f}(r)$ exactly as in (1.1) and (1.2). Equation (1.4) remains valid, where $\tilde{f}: \mathbf{C} \rightarrow \mathbf{C}^{n+1}-\{0\}$ is a representative of $f$. A nonzero linear functional $A$ on $\mathbf{C}^{n+1}$ determines the hyperplane $\{w: A(w)=0\} \subset \mathbf{C P}^{n}$, which we shall also denote by $A$. For such a hyperplane $A$, we let $n_{f}(A, r)$ denote the number of zeroes of $A \circ \tilde{f}$ (counted with multiplicities) in $\Delta(r)$, and we define $N(A, r)$ as in (1.5). It follows from Jensen's formula that

$$
N(A, r)=M_{r} \log |A \circ \tilde{f}|-M_{r_{0}} \log |A \circ \tilde{f}| .
$$

One also has the averaging formula

$$
T_{f}(r)=\int_{\mathbf{C P}^{n^{*}}} N(A, r) \omega^{n}(A),
$$

which generalizes (1.6). From (1.4) and (2.2), we obtain the First Main Theorem

$$
N(A, r)+m(A, r)=T_{f}(r)+O(1)
$$

where the proximity term $m(A, r)$ is given by

$$
m(A, r)=M_{r} \log (\|A\|\|\tilde{f}\| /|A \circ \tilde{f}|) \geqslant 0 .
$$

(We shall not mention the proximity term again.) Thus

$$
N(A, r) \leqslant T_{f}(r)+O(1) .
$$

We let $W$ denote the Wronskian of $\tilde{f}$,

$$
W=\left|\begin{array}{ccc}
\tilde{f}_{0} & \cdots & \tilde{f}_{n} \\
\tilde{f}_{0}^{\prime} & \cdots & \tilde{f}_{n}^{\prime} \\
\vdots & & \vdots \\
\tilde{f}_{0}^{(n)} & \cdots & \tilde{f}_{n}^{(n)}
\end{array}\right| \not \equiv 0,
$$

and we write

$$
R_{f}=\operatorname{Div} W \text {. }
$$

Thus

$$
N\left(R_{f}, r\right)=N_{W}(0, r) .
$$

In 1933, H. Cartan [7] obtained the Second Main Theorem

$$
(q-n-1) T_{f}(r) \leqslant \sum_{j=1}^{q} N\left(A_{j}, r\right)-N\left(R_{f}, r\right)+S_{f}(r),
$$

for any collection $\left\{A_{1}, \ldots, A_{q}\right\}$ of hyperplanes in general position. Cartan's Second Main Theorem (2.10) generalizes (1.9). Defining the defects exactly as in (1.10) one obtains immediately from (2.10) the Defect Relation 


$$
\sum_{j=1}^{q} \delta\left(A_{j}\right) \leqslant n+1
$$

for $A_{1}, \ldots, A_{q}$ as above.

In 1941, Ahlfors [2] proved (2.10) as well as similar inequalities for the "osculating curves", introducing an elegant geometric method that was later exploited by Carlson and Griffiths [6]. Other new proofs of (2.10) have been given by Cowen and Griffiths [10], by Chern [8] (for the case $n=2$ ) and by Yamaguchi [26]. We now give Cartan's proof, which uses only the Lemma on the Logarithmic Derivative and elementary linear algebra. In $\$ 5$ we shall give another new proof of (2.10) by making the map "equidimensional" and applying [6].

Let $f: \mathbf{C} \rightarrow \mathbf{C P}^{n}$ be as above and let $\tilde{f}: \mathbf{C} \rightarrow \mathbf{C}^{n+1}-\{0\}$ represent $f$. Let $q>n+1$, and let $A_{1}, \ldots, A_{q} \in\left(\mathbf{C}^{n+1}\right)^{*}$ represent hyperplanes in $\mathbf{C P}^{n}$ in general position; i.e., any $n+1$ of the $A_{j}$ are linearly independent. Let $g_{j}=A_{j} \circ \tilde{f}$ for $j=1, \ldots, q$. Thus, each $g_{j}$ is a linear combination of the $\tilde{f}_{i}$ $(0 \leqslant i \leqslant n)$, and each $\tilde{f}_{i}$ is a linear combination of any $n+1$ of the $g_{j}$. Note that

$$
\sum N\left(A_{j}, r\right)-N\left(R_{f}, r\right)=M_{r} \log \left|\frac{g_{1} \cdots g_{q}}{W}\right|+O(1) .
$$

Let $z \in \mathbf{C}$ be fixed. Permute the $g_{j}$ so that

$$
\left|g_{1}(z)\right| \leqslant\left|g_{2}(z)\right| \leqslant \cdots \leqslant\left|g_{q}(z)\right| \text {. }
$$

Suppose $W(z) \neq 0$. Then

$$
\frac{g_{1} \cdots g_{q}}{W}=\lambda\left|\begin{array}{ccc}
g_{1} \cdots g_{q} \\
\vdots & & \vdots \\
g_{1}^{(n)} & \cdots & g_{n+1}^{(n)}
\end{array}\right|=\lambda \frac{g_{n+2} \cdots g_{q}}{D} \text { at } z
$$

where

$$
D=\left|\begin{array}{lll}
1 & \cdots & 1 \\
g_{1}^{\prime} / g_{1} & \cdots & g_{n+1}^{\prime} / g_{n+1} \\
\vdots & & \vdots \\
g_{1}^{(n)} / g_{1} & \cdots & g_{n+1}^{(n)} / g_{n+1}
\end{array}\right|
$$

and

$$
\lambda=\operatorname{det}\left(A_{1} \ldots A_{n+1}\right) \in \mathbf{C}-\{0\} .
$$

Note that $\lambda$ depends only on the permutation of the $g_{j}$ (given by (2.13)). Since the $\tilde{f}_{i}$ are linear combinations of $g_{1}, \ldots, g_{n+1}$, we have by (2.13) the estimate

$$
\|\tilde{f}(z)\| \leqslant c\left|g_{n+1}(z)\right| \leqslant \cdots \leqslant c\left|g_{q}(z)\right|,
$$

where $c$ is independent of $z$. Therefore

$$
(q-n-1) \log \|\tilde{f}\| \leqslant \log \left|g_{n+2} \ldots g_{q}\right|+c^{\prime} \quad \text { at } z .
$$


Combining (2.14) and (2.16), we obtain

$$
(q-n-1) \log \|\tilde{f}\| \leqslant \log \left|\frac{g_{1} \cdots g_{q}}{W}\right|+\log |D|+c^{\prime \prime} \quad \text { at } z,
$$

where the constant $c^{\prime \prime}=c^{\prime}+\max \log 1 /|\lambda|$ is independent of $z$. We can replace the $g_{j}$ by the functions $\hat{g}_{j}=g_{j} / f_{0}$ in (2.15) without altering $D$. Thus

$$
\log |D| \leqslant \sum \log ^{+}\left|\hat{g}_{j}^{(i)} / \hat{g}_{j}\right|+\log (n+1) ! \quad(1 \leqslant j \leqslant q, 1 \leqslant i \leqslant n),
$$

and therefore

$$
(q-n-1) \log \|\tilde{f}\| \leqslant \log \left|\frac{g_{1} \cdots g_{q}}{W}\right|+\sum \log ^{+}\left|\hat{g}_{j}^{(i)} / \hat{g}_{j}\right|+O(1) .
$$

Averaging (2.17) over the circle $\{|z|=r\}$ and applying (1.4) and (2.12), we obtain

$$
(q-n-1) T_{f}(r) \leqslant \sum N\left(A_{j}, r\right)-N\left(R_{f}, r\right)+M_{r} \sum \log \left|\hat{g}_{j}^{(i)} / \hat{g}_{j}\right|+O(1) .
$$

The Second Main Theorem (2.10) then follows by applying the Lemma on the Logarithmic Derivative (1.13) and observing that

$$
T_{\hat{g}_{j}}(r) \leqslant T_{f}(r)+O(1) .
$$

As a consequence of the Defect Relation (2.11), one obtains Borel's theorem [3] that $f$ cannot omit $n+2$ hyperplanes in general position. Recently, M. Green [11] showed that $f$ cannot omit any set of $n+2$ distinct hyperplanes.

W. Stoll [23] showed in 1953 that if $f: \mathbf{C}^{n} \rightarrow \mathbf{C P}^{n}$ is a meromorphic map such that $f\left(\mathbf{C}^{m}\right)$ is not contained in a hyperplane of $\mathbf{C P}^{n}$ ( $m$ and $n$ arbitrary), then the Defect Relation (2.11) holds. Recently, A. Vitter [25] generalized Cartan's proof to yield Stoll's defect relation. The key step in Vitter's generalization is a "Lemma on the Logarithmic Partial Derivatives." (If $m \geqslant n$, then the result follows from Griffiths and King [16].)

3. Generalizations to several variables. We digress from the topic of this article, holomorphic curves, to discuss holomorphic and meromorphic maps from $\mathbf{C}^{n}$ into projective algebraic manifolds, which we shall later apply to the study of holomorphic curves. For further discussions of value distribution theory in several complex variables, see [27] and [29].

The first generalization of the Second Main Theorem to functions of several complex variables was Stoll's 1953 work [23] mentioned at the end of \$2. As in the theory of Cartan [7] and Ahlfors [2], Stoll's results concern the intersection of the image of $f$ with hyperplanes in $\mathbf{C P}^{n}$. In 1972, J. Carlson and P. Griffiths [6] achieved a major breakthrough by obtaining defect relations for hypersurfaces other than hyperplanes. A special case of the result of Carlson and Griffiths is that if $D_{1}, \ldots, D_{q}$ are smooth hypersurfaces of degree $d$ in $\mathbf{C P}^{n}$ with "normal crossings," and if $f: \mathbf{C}^{n} \rightarrow \mathbf{C P}^{n}$ is a holomorphic map of maximal rank, then

$$
\sum \delta\left(D_{j}\right) \leqslant(n+1) / d .
$$

(The definition of the defects $\delta\left(D_{j}\right)$ is given below.) We now give an outline of the Carlson-Griffiths theory. 
Let $M$ be a projective algebraic manifold, and let $L$ be a holomorphic line bundle on $M$. Suppose $L$ is given a hermitian metric $h$. The normalized curvature form $\eta \in c_{1}(L)$ of $h$ is defined by

$$
\eta=(\sqrt{-1} / 2 \pi) \partial \partial \bar{l} \log h(e, e),
$$

where $e$ is any nonvanishing local holomorphic section of $L$.

We let

$$
\begin{gathered}
\tilde{\omega}=(\sqrt{-1} / \pi) \partial \partial \bar{l} \log \|z\| \quad \text { on } \mathbf{C}^{n}-\{0\}, \\
l_{r}(z)=\min \left[\log ^{+}(r /\|z\|), \log \left(r / r_{0}\right)\right]
\end{gathered}
$$

for $z \in \mathbf{C}^{n}, r>r_{0}$ where $r_{0}$ is a fixed positive number, as before.

Let $f: \mathbf{C}^{n} \rightarrow M$ be a holomorphic map. Carlson and Griffiths [6] define the order function of $f$ with respect to $L$,

$$
T(L, r)=T_{f}(L, r)=\int_{\mathbf{C}^{n}} f^{*} \eta \wedge l_{r} \tilde{\omega}^{n-1} .
$$

(If $f: \mathbf{C} \rightarrow \mathbf{C P}^{n}$ and $L$ is the hyperplane section bundle with curvature form $\omega$, then $T_{f}(L, r)$ agrees with the order function $T_{f}(r)$ defined in \$2.) Definition (3.3) does not depend, modulo an $O(1)$ term, on the choice of the metric for $L$. Note that if $\eta$ is everywhere positive definite (in which case one says that $L$ is positive), then $T_{f}(L, r)$ is positive. Now suppose that $L$ is positive, and suppose that $D$ is a positive divisor on $M$ associated to $L$; i.e., $D$ is the divisor (zero-set with multiplicities) of a holomorphic section of $L$. One defines the counting function

$$
N(D, r)=\int_{f^{*} D} l_{r} \tilde{\omega}^{n-1}
$$

(where the multiplicities of the divisor $f^{*} D$ are taken into account when evaluating the integral), provided that the image of $f$ is not contained in the support of $D$. Identifying the set of positive divisors of $L$ with the projective space $\mathbf{P}(E)$, where $E=\Gamma(M, \vartheta(L))$, we have the averaging formula

$$
T(L, r)=\int_{\mathbf{P}(E)} N(D, r) \omega^{p}(D)+O(1)
$$

where $p=\operatorname{dim} E-1$. (See, for example, [20].) The Carlson-Griffiths First Main Theorem gives the inequality

$$
N(D, r) \leqslant T(L, r)+O(1) .
$$

The main result of Carlson and Griffiths [6] is the Second Main Theorem and defect relation for equidimensional maps, which we state below. A hypersurface $D$ is said to have normal crossings if locally $D$ is given as the zero locus of the product $z_{1} \ldots z_{k}(1 \leqslant k \leqslant \operatorname{dim} M)$ where $z_{1}, \ldots, z_{m}$ are local coordinates in $M$. We let $K_{M}$ denote the line bundle of holomorphic forms of top degree on $M$. We let $S_{f}(r)$ have the same meaning as in (1.8), with $T_{f}(r)$ replaced by $T_{f}(L, r)$, where $L$ is any positive line bundle on $M$. A map $f: \mathbf{C}^{n} \rightarrow M$, where $\operatorname{dim} M=n$, is said to be nondegenerate if $f$ has rank $n$ at some point of $\mathbf{C}^{n}$; if $f$ is nondegenerate, we let $R_{f}$ denote the divisor of the Jacobian determinant of $f$. With this notation in mind, we can now state 
the Carlson-Griffiths Second Main Theorem:

(3.7) THEOREM [6]. Let $f: \mathbf{C}^{n} \rightarrow M$ be a nondegenerate holomorphic map, where $M$ is an n-dimensional compact complex manifold. Let $L$ be a positive line bundle on $M$, and let $D$ be a positive divisor associated to $L$. Suppose that $D$ is the union of smooth hypersurfaces (with multiplicity one) and $D$ has normal crossings. Then

$$
T_{f}(L, r)+T_{f}\left(K_{M}, r\right) \leqslant N(D, r)-N\left(R_{f}, r\right)+S_{f}(r) .
$$

Combining (3.6) and (3.7), we obtain the inequality

$$
T_{f}\left(K_{M}, r\right) \leqslant S_{f}(r) \text {. }
$$

Since (3.8) is contradictory if $K_{M}$ is positive, we have the following consequence of (3.7):

(3.9) COROllaRY [6]. If $M$ is an n-dimensional compact complex manifold with $K_{M}$ positive, then there do not exist any nondegenerate holomorphic maps $f: \mathbf{C}^{n} \rightarrow M$.

Theorem (3.7) also yields defect relations. As before, we define the defect

$$
\delta(D)=\lim \inf \left[1-\frac{N(D, r)}{T(L, r)}\right],
$$

and note that $0 \leqslant \delta(D) \leqslant 1$. Let

$$
\lambda(L)=\inf \left\{t \in \mathbf{R}: t c_{1}(L)+c_{1}\left(K_{M}\right) \geqslant 0\right\},
$$

where $\gamma \geqslant 0$, for $\gamma \in H^{2}(M, \mathbf{R})$, means that $\gamma$ can be represented by an everywhere positive-semi-definite $(1,1)$-form on $M$. (Carlson and Griffiths [6] use the notation $\lambda(L)=\left[c_{1}\left(K_{M}^{*}\right) / c_{1}(L)\right]$. If $H^{2}(M, \mathbf{R}) \approx \mathbf{R}$, then $\lambda(L)=$ $c_{1}\left(K_{M}^{*}\right) / c_{1}(L)$.) Suppose $D_{1}, \ldots, D_{q}$ are smooth hypersurfaces with normal crossings such that each $D_{j}$ is the divisor of a section of $L$. Applying Theorem (3.7) to the line bundle $L^{q}$, and using the fact that $[\lambda(L)+\varepsilon] T(L, r)+$ $T\left(K_{M}, r\right) \geqslant O(1)$ for $\varepsilon>0$, one obtains the Carlson-Griffiths Defect Relations

$$
\sum_{j=1}^{q} \delta\left(D_{j}\right) \leqslant \lambda(L)
$$

For example, let $M=\mathbf{C P}^{n}$, and let $L=L_{H}^{d}$, where $L_{H}$ is the hyperplane section bundle on $\mathbf{C P}^{n}$. Then $H^{2}\left(\mathbf{C P}^{n}, Z\right) \approx Z, c_{1}\left(L_{H}\right)=1, c_{1}\left(K_{\mathbf{C P p}^{n}}^{*}\right)=n+$ 1. Thus $\lambda\left(L_{H}^{d}\right)=(n+1) / d$, and we obtain the defect relation (3.1). (The case $d=1$ of (3.1) gives Stoll's defect relation for equidimensional holomorphic maps.)

The distribution of values of a holomorphic map $f: \mathbf{C}^{n} \rightarrow \mathbf{C P}^{n}$ with respect to algebraic submanifolds of $\mathbf{C P}^{n}$ of codimension greater than one is an entirely different story. For example, the Fatou-Bieberbach map $f: \mathbf{C}^{2} \rightarrow \mathbf{C}^{2}$ omits a (rather large) open set, so there are no defect relations with respect to points in $\mathbf{C P}^{2}$. Furthermore, $f^{-1}$ \{point may grow much faster than $f$ (for $n \geqslant 2$ ), so the First Main Theorem is not valid for codimension greater than one (see [9]).

The Second Main Theorem (3.7) and the Defect Relations (3.12) have been extended in [16] to the case where $C^{n}$ is replaced by any affine algebraic 
manifold $V$ with $\operatorname{dim} V \geqslant n=\operatorname{dim} M$, and in [21] where $f$ is allowed to be a meromorphic map and $D$ can have singularities other than normal crossings. If $D$ has singularities, then there is an extra term in (3.7) and the defect relations are of the form $\Sigma \delta\left(D_{j}\right) \leqslant \lambda(L)+s(L, D)$ where $s(L, D)$ depends only on $L$ and the algebraic structure of the singularities of the divisor $D=\cup D_{j}$. In particular, it follows from [2, pp. 173-175] that (3.7) remains valid when $f$ is meromorphic and $D$ is any divisor of $L$ with only normal crossing singularities (e.g., a component of $D$ may intersect itself normally). Thus, a nondegenerate meromorphic map from $\mathbf{C}^{n}$ to $\mathbf{C P}^{n}$ cannot omit a hypersurface of degree greater than $n+1$ with only normal crossing singularities.

4. Open problems on holomorphic curves. One of the major open problems in value distribution theory is whether the Carlson-Griffiths Defect Relations (3.12) for equidimensional maps hold for holomorphic curves. Conjecture I below restates this question, which has been previously asked by P. Griffiths [15] and M. Green.

A holomorphic curve $f: \mathbf{C} \rightarrow M$ in an algebraic manifold $M$ is said to be algebraically nondegenerate if $f(\mathbf{C})$ is not contained in any algebraic hypersurface of $M$.

CONJeCtURE I. Let $L$ be a positive line bundle on a projective algebraic manifold $M$, and let $D_{1}, \ldots, D_{q}$ be smooth multiplicity-one divisors of $L$ with normal crossings. Let $f: \mathbf{C} \rightarrow M$ be an algebraically nondegenerate holomorphic curve. Then, using the notation of $\$ 3$,

$$
\sum \delta\left(D_{j}\right) \leqslant \lambda(L)
$$

The following two conjectures are special cases of Conjecture I.

CONJeCTURE Ia. Let $M$ be a projective algebraic manifold with $K_{M}$ positive. Then there are no algebraically nondegenerate holomorphic curves in $M$.

CONJECTURE Ib. Let $f: \mathbf{C} \rightarrow \mathbf{C P}^{n}$ be an algebraically nondegenerate holomorphic curve, and let $D_{1}, \ldots, D_{q}$ be smooth hypersurfaces in $\mathbf{C P}^{n}$ of degree $d$, such that $D_{1} \cup \cdots \cup D_{q}$ has normal crossings. Then

$$
\sum \delta\left(D_{j}\right) \leqslant(n+1) / d .
$$

Conjecture Ia for the case $\operatorname{dim} M=1$ follows from Liouville's Theorem and the fact that the compact complex curves of genus greater than 1 are uniformized by the disk. The case $d=1$ of Conjecture Ib is Cartan's Defect Relation (2.11).

A consequence of Conjecture I would be the Picard-type Theorem that an algebraically nondegenerate holomorphic curve in $M$ cannot omit any divisor of $L$ if $\lambda(L)<1$, or equivalently if $K_{V} \otimes L$ is positive. For example, if $\boldsymbol{M}=\mathbf{C P}^{n}$ this Picard-type Theorem can be stated as follows:

CONJECTURE Ic. Let $D$ be a hypersurface with normal crossings in $\mathbf{C P}^{n}$ of degree at least $n+2$. Then there are no algebraically nondegenerate holomorphic curves $f: \mathbf{C} \rightarrow \mathbf{C P}^{n}-D$.

Some special cases of Conjectures Ia and Ic have been studied by $\mathbf{M}$. 
Green [12], [14]. Let $V_{d}$ denote the "Fermat variety" of degree $d$,

$$
\left\{w \in \mathbf{C P}^{n}: w_{0}^{d}+w_{1}^{d}+\cdots+w_{n}^{d}=0\right\} .
$$

Suppose $f: \mathbf{C} \rightarrow V_{d}$ is a holomorphic curve. M. Green [14] proved that $f(\mathbf{C})$ lies in a hyperplane section of $V_{d}$ if $d>n^{2}-1$. Conjecture Ia would imply that $f$ is algebraically degenerate if $d>n+1$. Now suppose we are given a holomorphic curve $g: \mathbf{C} \rightarrow \mathbf{C P}^{n}-V_{d}$. M. Green [14] proved that $g(\mathbf{C})$ lies in a projective hyperplane if $d>n(n+1)$. Conjecture Ic would imply that $g$ is algebraically degenerate if $d>n+1$. N. Toda [24] also studied maps $g: \mathbf{C} \rightarrow$ $\mathbf{C P}^{n}-V_{d}$ and obtained a similar result.

EXAMPLe ([14, p. 74] OR [13, p. 7]). Let $f: \mathbf{C} \rightarrow \mathbf{C P}^{2}-V_{4}$ be given by

$$
f_{0}(z)=1+e^{4 z}, \quad f_{1}(z)=\sqrt[4]{-1}\left(1-e^{4 z}\right), \quad f_{2}(z)=\sqrt[4]{-8} e^{z} .
$$

Then $f(\mathbf{C})$ is contained in the irreducible quartic

$$
2 w_{2}^{4}+\left(w_{0}+w_{1} / \sqrt[4]{-1}\right)^{3}\left(w_{0}-w_{1} / \sqrt[4]{-1}\right)=0 .
$$

This example shows that the conclusion of Conjecture Ic is false if one assumes only that $f(\mathbf{C})$ does not lie in a hyperplane.

A question which naturally arises from the above example is whether any holomorphic curve in $\mathbf{C P}^{n}-D$, where $D$ is given as in Conjecture Ic, must lie in an algebraic hypersurface of degree at most equal to the degree of $D$. The answer to this question is unknown. However, the following new example constructed by $\mathrm{M}$. Green and R. Molzon suggests that the answer to this question probably is "no":

EXAMPLE. Consider the homogeneous polynomials

$$
\begin{aligned}
& Q_{1}=w_{1} w_{3}+a\left(w_{0} w_{3}-w_{2}^{2}\right), \quad Q_{2}=w_{0} w_{2}+a w_{1}^{2}, \\
& R_{1}=w_{1}^{3}+w_{3}^{3}, \quad R_{2}=w_{0}^{3}+w_{2}^{3},
\end{aligned}
$$

where $a$ is a complex constant. Let $M$ be the algebraic hypersurface in $\mathbf{C P}^{3}$ given by the equation

$$
Q_{1} R_{1}+Q_{2} R_{2}=0 \text {. }
$$

One can easily check that $M$ is nonsingular if $a=0$. Therefore, $M$ is nonsingular for sufficiently small $a$. (In fact, $M$ is singular for only finitely many values of $a$.) Choose $a \neq 0$ such that $M$ is nonsingular, and let $f: \mathbf{C} \rightarrow \mathbf{C P}^{3}$ be given by

$$
f_{0}(z)=1+z, \quad f_{1}(z)=a z(1+z), \quad f_{2}(z)=-a^{3} z^{2}(1+z), \quad f_{3}(z)=a^{6} z^{4} .
$$

Since $Q_{1} \circ f=Q_{2} \circ f=0$, it follows that $f$ is a holomorphic curve in $M$. Let $L=L_{H} \mid M$, where $L_{H}$ is the hyperplane section bundle in $\mathbf{C P}^{3}$. We note that $K_{M}=L^{\operatorname{deg} M-4}=L$, and thus $\lambda(L)=-1$. Therefore, the conclusion of Conjecture I does not hold in this case for any collection of divisors of $L$. Of course, the curve $f$ is algebraically degenerate, $f(\mathbf{C})$ being contained in a divisor of $L^{2}$, but not in a divisor of $L$. Thus Conjecture $\mathrm{I}$ is false in general if we replace algebraic nondegeneracy by the requirement that $f(\mathbf{C})$ does not lie in a divisor of $L$. (However, I do not know of any such counterexample with $\lambda(L)$ positive.) This example also shows that if $K_{M}$ is positive as in Conjecture Ia, then the holomorphic curves in $M$ do not necessarily lie in divisors of $K_{M}$. 
Suppose that $M$ is an algebraic surface imbedded in some $\mathbf{C P}^{n}$ and let $L$ be a positive line bundle on $M$. It is not known if there is a bound on the degree of $f(\mathbf{C})$, where $f$ is an algebraically degenerate holomorphic curve such that the defect relation of Conjecture I fails. In particular, Conjecture Ia suggests the following question:

Problem. Let $M$ be a projective algebraic surface such that $K_{M}$ is positive. Is there only a finite number of rational and elliptic curves in $M$ ?

This problem could perhaps best be attacked by transcendental methods.

We can instead assume that $M$ contains no rational or elliptic curves, and state in place of Conjecture Ia:

CONJeCtURe II. Let $M$ be a projective algebraic manifold that contains no rational or elliptic curves. Then there are no holomorphic curves in $M$.

The hyperbolic manifolds (see [17]) have the property that they contain no holomorphic curves. A result of Brody's thesis is that the converse is true for compact manifolds:

THEOREM ( $\mathrm{R}$. BRODY [4]). Let $M$ be a compact complex manifold. Then $M$ is hyperbolic if and only if $M$ contains no holomorphic curves.

Thus, Conjecture II is equivalent to the statement that a projective algebraic manifold is hyperbolic if and only if it contains no rational or elliptic curves. The problem of describing the hyperbolic algebraic manifolds of dimension $\geqslant 2$ is quite difficult. (A compact complex curve is hyperbolic if and only if its genus is greater than 1.) Brody and Green [5] recently constructed a one-parameter family $\left\{M_{t}\right\}$ of algebraic hypersurfaces in $\mathbf{C P}^{3}$ such that $M_{t}$ is hyperbolic for $t \neq 0$ but $M_{0}$ is not hyperbolic $\left(M_{0}=V_{2 d}\right.$, $d \geqslant 25$ ). Their example answers two old questions. First, it provides simplyconnected compact hyperbolic manifolds, which were conjectured not to exist. Second, it shows that hyperbolicity is not a topological invariant, contrary to an assertion of Bloch.

A result similar to Conjecture Ia was recently obtained by T. Ochiai. Let $M$ be an $n$-dimensional projective algebraic manifold. Ochiai [28] proved that if the space of holomorphic one-forms on $M$ has dimension greater than $n$ and if the Albanese map of $M$ is an imbedding, then every holomorphic curve in $M$ is algebraically degenerate.

5. Yet another proof of the Cartan-Ahlfors theorem. We use the results of Carlson and Griffiths [6] to give a new geometric proof of the Second Main Theorem (2.10). This proof arose from an unsuccessful attempt to prove Conjecture I.

We first prove Theorem (2.10) for the case where $f: \mathbf{C} \rightarrow \mathbf{C P}{ }^{n}$ is a finite order map (and $f(\mathbf{C})$ is not contained in a hyperplane). Choose a representation $\tilde{f}: \mathbf{C} \rightarrow \mathbf{C}^{n+1}-\{0\}$ of $f$ such that the $\tilde{f}_{j}$ are of finite order. (For example, let $\phi$ be a Weierstrass product such that $\operatorname{Div} \phi=f^{*} \operatorname{Div} w_{0}$, and let $\tilde{f}_{j}=$ $\phi f^{*}\left(w_{j} / w_{0}\right)$.) We shall construct a meromorphic map $F$ from $\mathbf{C}^{1} \times \mathbf{C P}^{n-1}$ into $\mathbf{C P}^{n}$ such that $F(t,(1: 0: \ldots: 0))=f(t)$, and such that the order and counting functions of $F$ are roughly equal to those of $f$. Let $\tilde{F}: \mathbf{C}^{1} \times \mathbf{C}^{n} \rightarrow \mathbf{C}^{n+1}$ be given by 


$$
\tilde{F}\left(t, z_{0}, z_{1}, \ldots, z_{n-1}\right)=z_{0} \tilde{f}(t)+z_{1} \tilde{f}^{\prime}(t)+\cdots+z_{n-1} \tilde{f}^{(n-1)}(t) .
$$

The map $F$ is then defined by the commutative diagram of meromorphic maps

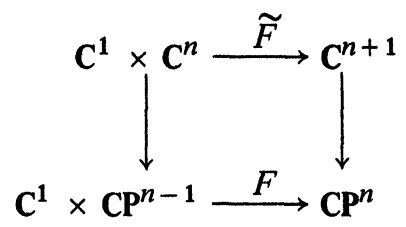

where the vertical arrows are the natural (meromorphic) maps. Let $R_{F}$ denote the divisor of the Jacobian determinant of $F$. One easily verifies the identity

$$
R_{F}=R_{f} \times \mathrm{CP}^{n-1}+\mathrm{C}^{1} \times H,
$$

where $R_{f}$ is given by (2.8) and $H$ is the hyperplane $\left\{z_{n-1}=0\right\}$. Let

$$
l_{r}(t, z)=l_{r}(t), \quad \beta=(\sqrt{-1} / 2 \pi) d t \wedge d \bar{t}+\omega^{\prime}
$$

on $\mathbf{C}^{1} \times \mathbf{C P}^{n-1}$, where $\omega^{\prime}$ is the normalized Kähler form (recall (2.1)) of $\mathbf{C P}^{n-1}$. For a divisor $D$ on $\mathbf{C}^{1} \times \mathbf{C P}^{n-1}$, we write

$$
\tilde{N}(D, r)=\int_{D} l_{r} \beta^{n-1} \text {. }
$$

We define the counting function for a hyperplane $A$ in $\mathbf{C P}^{n}$ by

$$
\tilde{N}(A, r)=\tilde{N}\left(f^{*} A, r\right) .
$$

It follows from (5.2) and (5.3) that

$$
\tilde{N}\left(R_{F}, r\right)=N\left(R_{f}, r\right)+\gamma(r),
$$

where

$$
\gamma(r)=(n-1) \frac{\sqrt{-1}}{2 \pi} \int_{\mathbf{C}} l_{r}(t) d t \wedge d \bar{t}=\frac{n-1}{2}\left(r^{2}-r_{0}^{2}\right) .
$$

We similarly define the order function

$$
\tilde{T}(r)=\tilde{T}_{F}(r)=\int F^{*} \omega \wedge l_{r} \beta^{n-1},
$$

where $\omega$ is given by (2.1). (The form $F^{*} \omega$ has locally integrable coefficients on $\mathbf{C}^{\mathbf{l}} \times \mathbf{C P}^{n-1}$, and of course is $\mathbf{C}^{\infty}$ where $F$ is holomorphic. See [21, p. 161].) We shall use the notation

$$
\operatorname{Ave}_{z} \phi(z)=\int_{\mathbf{C P}^{n-1}} \phi \omega^{n-1}
$$

for a function $\phi$ on $\mathbf{C P}^{n-1}$. We let $F_{z}: \mathbf{C} \rightarrow \mathbf{C P}^{n}$ be given by $F_{z}(t)=F(t, z)$, for each $z \in \mathbf{C P}^{n}$. Note that $F_{(1: 0: \ldots: 0)}=f$. We write

$$
T_{z}(r)=T_{F_{z}}(r), \quad N_{z}(A, r)=N_{F_{z}}(A, r) .
$$

(5.8) Lemma. $\tilde{T}(r)=\operatorname{Ave}_{z} T_{z}(r)+\gamma(r)$.

Proof. Let 


$$
\hat{F}(t, z)=\hat{F}_{z}(t)=\tilde{f}(t)+\frac{z_{1}}{z_{0}} \tilde{f}^{\prime}(t)+\cdots+\frac{z_{n-1}}{z_{0}} \tilde{f}^{(n-1)}(t),
$$

regarded as an $(n+1)$-tuple of meromorphic functions (for $t \in \mathbf{C}, z \in$ $\left.\mathbf{C P}^{n-1}\right)$. Note that for almost all $z \in \mathbf{C P}^{n-1}, F_{z}$ has values in $\mathbf{C}^{n+1}-\{0\}$ and is a representative of $F_{z}$. From the identity of currents

$$
(\sqrt{-1} / \pi) \partial \check{\partial} \log \|\hat{F}\|=F^{*} \omega-\operatorname{div} z_{0}
$$

(see [21, Lemma 2.6] and [6, 1.12]), we conclude that

$$
\tilde{T}(r)=\left(\frac{\sqrt{-1}}{\pi} \partial \bar{\partial} \log \|\hat{F}\|, l_{r} \beta^{n-1}\right)+\gamma(r) .
$$

Evaluating the pairing in the above formula, one obtains

$$
\left(\frac{\sqrt{-1}}{\pi} \partial \bar{\partial} \log \|\hat{F}\|, l_{r} \beta^{n-1}\right)=\int \partial \bar{\partial} \log \|\hat{F}\| \wedge l_{r} \omega^{\prime n-1}=\operatorname{Ave}_{z} T_{z}(r)
$$

(where the integral is over those points of $\mathbf{C}^{1} \times\left(\mathbf{C P}^{n}-\left\{z_{0}=0\right\}\right)$ where $\hat{F} \neq 0$ ), which proves the lemma.

Using the identity

$$
(\sqrt{-1} / \pi) \partial \bar{\partial} \log |A \circ \hat{F}|=F^{*} \operatorname{Div} A-\operatorname{Div} z_{0}
$$

for $A \in\left(C^{n+1}\right)^{*}$, one can repeat the above proof to obtain the following analogue of Lemma (5.8) for the counting function:

(5.12) Lemma. $\tilde{N}(A, r)=$ Ave $_{z} N_{z}(A, r)+\gamma(r)$, for any hyperplane $A \subset$ $\mathbf{C P}^{n}$.

One can show that the First Main Theorem

$$
\tilde{N}(A, r) \leqslant \tilde{T}(r)+O(1)
$$

(where $A$ is a projective hyperplane) is valid for our modified order and counting functions. (This F.M.T. is needed only for the proof of the S.M.T. below.) Let $\left\{A_{1}, \ldots, A_{q}\right\}$ be a collection of hyperplanes in general position. Using the methods of Carlson and Griffiths [6], we obtain the Second Main Theorem

$$
(q-n-1) \tilde{T}(r) \leqslant \sum_{j=1}^{q} \tilde{N}\left(A_{j}, r\right)-N\left(R_{F}, r\right)-n \gamma(r)+S_{F}(r) .
$$

Proof of (5.13). (This proof assumes familiarity with [6], [16] or [21] and can be omitted without loss of continuity.) We consider the Carlson-Griffiths singular volume form $\Psi$ on $\mathbf{C P}^{n}$ (see p. 566 of [6]) that blows up along the hypersurface $D=A_{1} \cup \cdots \cup A_{q}$. We let $F^{*} \Psi=\xi \beta^{n}$. The proof of Lemma 3.7 in [21] (which extends Lemma 1.14 in [6] or Lemma 6.20 in [16] to meromorphic mappings) yields the current identity

$$
(\sqrt{-1} / 2 \pi) \partial \overline{\log } \xi+\operatorname{Ric} \beta^{n}=F^{*} \operatorname{Ric} \Psi-F^{*} D+R_{F} .
$$

Note that (5.14) contains the extra term Ric $\beta^{n}$, and that

$$
\operatorname{Ric} \beta^{n}=-n \omega^{\prime} \text {. }
$$

The Second Main Theorem (5.13) follows from (5.14) as in the proof of the 
S.M.T. in [6], [15], or [21, pp. 168-169], except in place of $\mu(r)$ we define

$$
\tilde{\mu}(r)=\frac{1}{2} M_{r} \text { Ave }_{z} \log \xi(t, z) .
$$

(The extra term $-n \gamma(r)$ in (5.13) comes from the term Ric $\beta^{n}$ in (5.14).)

Combining (5.4), (5.8), (5.12), and the Second Main Theorem (5.13), we obtain the inequality

$$
(q-n-1) \operatorname{Ave}_{z} T_{z}(r) \leqslant \sum_{j=1}^{q} \operatorname{Ave}_{z} N_{z}\left(A_{j}, r\right)-N\left(R_{f}, r\right)+S_{F}(r) .
$$

The Second Main Theorem (2.10) is a consequence of (5.15), (5.8) and the following two estimates:

$$
\begin{aligned}
& \text { Lemma. }\left|T_{f}(r)-\operatorname{Ave}_{z} T_{z}(r)\right| \leqslant S_{f}(r) . \\
& \text { Lemma. }\left|N_{f}(A, r)-\operatorname{Ave}_{z} N_{z}(A, r)\right| \leqslant S_{f}(r) .
\end{aligned}
$$

(Recall that $S_{f}(r) \leqslant O(\log r)$ when $f$ is of finite order.) We shall use the following inequality in the proof of (5.16) and (5.17): Suppose $g$ is a meromorphic function on $\mathbf{C}$, and let

$$
g_{z}=g+\left(z_{1} / z_{0}\right) g^{\prime}+\cdots+\left(z_{n-1} / z_{0}\right) g^{(n-1)},
$$

for each $z=\left(z_{0}: \ldots: z_{n-1}\right) \in \mathbf{C P}^{n-1}$ with $z_{0} \neq 0$. Then

$$
0 \leqslant \text { Ave }_{z} \log \left|g_{z}\right|-\log |g| \leqslant \sum_{i=1}^{n-1} \log ^{+}\left|g^{(i)} / g\right|+\frac{1}{2} \log n .
$$

To verify (5.18), we note that for $w=\left(w_{0}, \ldots, w_{n-1}\right) \in \mathbf{C}^{n}-\{0\}$, we have

$$
\operatorname{Ave}_{z} \log \left(|z \cdot w| /\left|z_{0}\right|\right)=\log \|w\| \leqslant \sum_{j=0}^{n-1} \log ^{+}\left|w_{j}\right|+\frac{1}{2} \log n .
$$

The inequality (5.18) follows from (5.19) with $w_{j}=g^{(j)} / g$ (for $0 \leqslant j \leqslant$ $n-1)$.

To prove (5.17), we let $A \in\left(\mathbf{C}^{n+1}\right)^{*}$ represent a hyperplane in $\mathbf{C P}^{n}$, and we let $g=A \circ \tilde{f}$. Then $g_{z}=A \circ \hat{F}_{z}$. Inequality (5.17) follows by averaging (5.18) over the circle $\{|t|=r\}$ and applying (2.2) and the Lemma of the Logarithmic Derivative (1.13).

We now verify (5.16): We note that

$$
\begin{aligned}
\log \|\tilde{f}\| & =\operatorname{Ave}_{w} \log \left(|\tilde{f} \cdot w| /\left|w_{0}\right|\right) \leqslant \operatorname{Ave}_{w} \operatorname{Ave}_{z}\left(\left|\hat{F}_{z} \cdot w\right| /\left|w_{0}\right|\right) \\
& =\operatorname{Ave}_{z} \log \left\|\hat{F}_{z}\right\| .
\end{aligned}
$$

$\left(z \in \mathbf{C P}^{n-1}, w \in \mathbf{C P}^{n}\right.$.) The inequality in (5.20) follows from the first inequality in (5.18) applied to the function $g=w_{0}^{-1}(\tilde{f} \cdot w)$, and the equalities in (5.20) follow from (5.19). It then follows from (1.4) that $T_{f}(r) \leqslant \mathrm{Ave}_{z} T_{z}(r)+$ $O(1)$. To obtain the reverse inequality we note that

$$
\begin{aligned}
\log \left\|\hat{F}_{z}\right\| & \leqslant \max _{j} \log \left|\left(\hat{F}_{z}\right)_{j}\right|+\log (n+1) \\
& \leqslant \log \|\tilde{f}\|+\max _{j} \log \left|\left(\hat{F}_{z}\right)_{j} / \tilde{f}_{j}\right|+\log (n+1) \quad(0 \leqslant j \leqslant n) .
\end{aligned}
$$

Averaging over $z$ and applying (5.18) with $g=\tilde{f}_{j}$, we then conclude that 


$$
\text { Ave }_{z} \log \left\|\hat{F}_{z}\right\| \leqslant \log \|\tilde{f}\|+\sum_{j, i} \log ^{+}\left|\tilde{f}_{j}^{(i)} / \tilde{f}_{j}\right|+\log (n+1)+\frac{1}{2} \log n
$$

$(0 \leqslant j \leqslant n, 1 \leqslant i \leqslant n-1)$. Applying (1.4) and (1.13), we obtain

$$
\text { Ave }_{z} T_{z}(r) \leqslant T_{f}(r)+S_{f}(r) \text {, }
$$

which completes the proof of (5.16) and thus of Theorem (2.10) for the case where $f$ has finite order.

We now briefly indicate how the proof is modified for the case where $f$ is of infinite order. In this case, we let $f^{\#}: \mathbf{C} \rightarrow \mathbf{C}^{n+1}-\{0\}$ be an arbitrary representation of $f$, and we let

$$
\tilde{f}=\left(1 / f_{0}^{\#}\right) f^{\#}
$$

be regarded as an $(n+1)$-tuple of meromorphic functions. Let $E=$ Support(Div $f_{0}^{\#}$ ), and choose an entire function $\phi$ such that Div $\phi=E$ (with multiplicity 1). We let $\psi=f_{0}^{\#} \phi^{n-1}$, and define $\tilde{F}: \mathbf{C}^{1} \times \mathbf{C}^{n} \rightarrow \mathbf{C}^{n+1}$ by

$$
\tilde{F}(t, z)=\psi(t)\left[z_{0} \tilde{f}(t)+z_{1} \tilde{f}^{\prime}(t)+\cdots+z_{n-1} \tilde{f}^{(n-1)}(t)\right],
$$

which induces $F$ as before. We obtain the identity

$$
R_{F}=R_{f} \times \mathbf{C P}^{n-1}+\mathbf{C}^{1} \times H+\left(n^{2}-1\right) E \times \mathbf{C P}^{n-1}
$$

in place of (5.2). The proof then proceeds as before except we subtract the quantity $\left(n^{2}-1\right) N(E, r)$ from the right-hand side of (5.15). Also, in Lemmas (5.16) and (5.17) we replace the term $T_{f}(r)$ by $T_{f}(r)+(n-1) N(E, r)$, and $N_{f}(A, r)$ by $N_{f}(A, r)+(n-1) N(E, r)$. To prove the revised Lemmas (5.16) and (5.17) we need to use the fact that

$$
T_{\tilde{f}_{j}}(r) \leqslant T_{f}(r)+O(1)
$$

and hence $S_{f_{j}}(r) \leqslant S_{f}(r)$.

In order to generalize this proof to apply to Conjecture I, one would need to construct $F$ such that Lemmas (5.8), (5.12), (5.16), and (5.17) hold. This appears to be a formidable task.

\section{REFERENCES}

1. L. V. Ahlfors, Beiträge zur Theorie der meromorphen Funktionen, C. R. 7 Congr. Math. Scand., Oslo, 1929, pp. 84-88.

2. , The theory of meromorphic curves, Acta Soc. Sci. Fenn. Nova Ser. A 3 (1941), no. 4, 1-31. MR 2, 357.

3. E. Borel, Sur les zéros des fonctions entières, Acta Math. 20 (1897), 357-396.

4. R. Brody, Compact manifolds and hyperbolicity (to appear).

5. R. Brody and M. L. Green, $A$ family of smooth hyperbolic hypersurfaces in $\mathbf{P}_{\mathbf{3}}$ (to appear).

6. J. Carlson and P. A. Griffiths, $A$ defect relation for equidimensional holomorphic mappings between algebraic varieties, Ann. of Math. (2) 95 (1972), 557-584. MR 47 \# 497.

7. H. Cartan, Sur les zéros des combinaisons linéaires de $p$ fonctions holomorphes données Mathematica (Cluj) 7 (1933), 5-31.

8. S. S. Chern, Holomorphic curves in the plane, Differential geometry (in honor of Kentaro Yano), Kinokuniya Book-Store Co., Tokyo, 1972, pp. 73-94. MR 48 \#2941.

9. M. Cornalba and B. Shiffman, A counterexample to the "Transcendental Bézout problem", Ann. of Math. (2) 96 (1972), 402-406. MR 47 \#499.

10. M. Cowen and P. A. Griffiths, Holomorphic curves and metrics of negative curvature, J. Analyse Math. (to appear).

11. M. L. Green, Holomorphic maps into complex projective space omitting hyperplanes, Trans. Amer. Math. Soc. 169 (1972), 89-103. MR 46 \# 7547. 
12. M. L. Green, On the functional equation $f^{2}=e^{2 \varphi 1}+e^{2 \varphi_{2}}+e^{2 \varphi 3}$ and a new Picard theorern, Trans. Amer. Math. Soc. 195 (1974), 223-230. MR 50 \#10.

13. - Some examples and counter-examples in value distribution theory for several variables, Compositio Math. 39 (1975), 317-322. MR 51 \#10698.

14. - Some Picard theorems for holomorphic maps to algebraic varieties, Amer. J. Math. 97 (1975), 43-75. MR 51 \#3544.

15. P. A. Griffiths, Holomorphic mappings: Survey of some results and discussion of open problems, Bull. Amer. Math. Soc. 78 (1972), 374-382. MR 45 \#3786.

16. P. A. Griffiths and J. King, Nevanlinna theory and holomorphic mappings between algebraic varieties, Acta Math. 130 (1973), 145-220.

17. S. Kobayashi, Hyperbolic manifolds and holomorphic mappings, Dekker, New York, 1970. MR 43 \#3503.

18. R. Nevanlinna, Le théorème de Picard-Borel et la théorie des fonctions meromorphes, Gauthiers-Villars, Paris, 1929.

19. , Analytic functions, Springer-Verlag, Berlin, 1970. MR 43 \# 5003.

20. B. Shiffman, Applications of geometric measure theory to value distribution theory for meromorphic maps, Value-distribution theory, Part A, Dekker, New York, 1974, pp. 63-95. MR $51 \# 13295$.

21. __ Nevanlinna defect relations for singular divisors, Invent. Math. 31 (1975), 155-182.

22. T. Shimizu, On the theory of meromorphic functions, Japan. J. Math. 6 (1929), 119-171.

23. W. Stoll, Die beiden Hauptsätze der Wertverteilungstheorie bei Funktionen mehrerer komplexer Veränderlichen. I, II, Acta Math. 90 (1953), 1-115; ibid. 92 (1954), 55-169. MR 17, 893, 894.

24. N. Toda, On the functional equation $\sum_{i=0}^{p} a_{i} f_{i}^{n_{i}}=1$, Tôhoku Math. J. 23 (1971), 289-299. MR 45 \#551.

25 A. Vitter, The lemma of the logarithmic derivative in several complex variables, Duke Math. J. (to appear).

26. H. Yamaguchi, Defect relations of holomorphic curves in complex projective space, 1972 (unpublished manuscript).

27. P. A. Griffiths, Entire holomorphic mappings in one and several complex variables, Ann. of Math. Studies, no. 85, Princeton Univ. Press, Princeton, N. J., 1976.

28. T. Ochiai, On holomorphic curves in algebraic varieties with ample irregularity (to appear).

29. W. Stoll, Aspects of value distribution theory in several complex variables, Bull. Amer. Math.

Soc. 83 (1977), 166-183.

School of Mathematics, Institute for Advanced Study, Princeton, New Jersey 08540

Department of Mathematics, Johns Hopkins University, Baltimore, Maryland 21218 